FL

72.99

M78

1978 


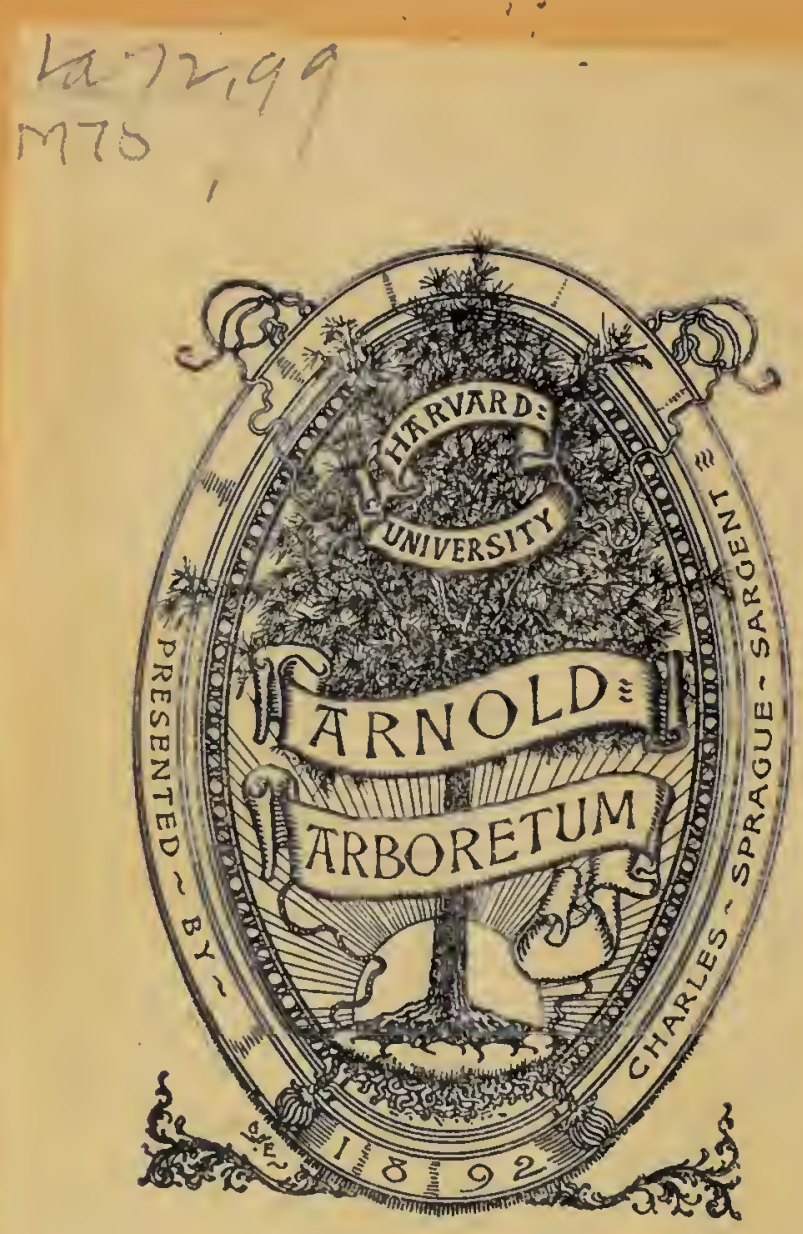




\section{A LIST OF PLANTS}

COLLECTED IN BERMUDA IN 1905

BY

ALBERT HANFORD MOORE

Includina a Description of Two New Species

CAMBRIDGE, MASSACHUSETTS

Issued March 12, 1906 
Q -

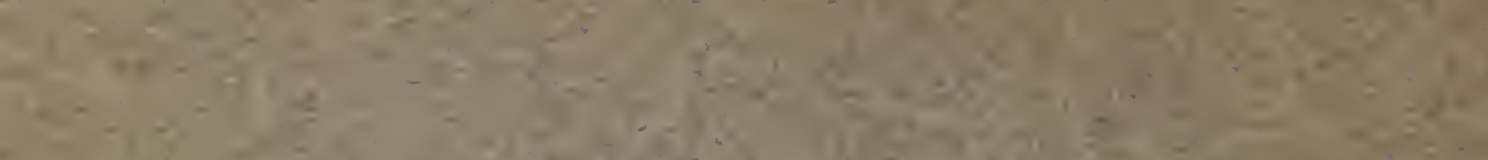

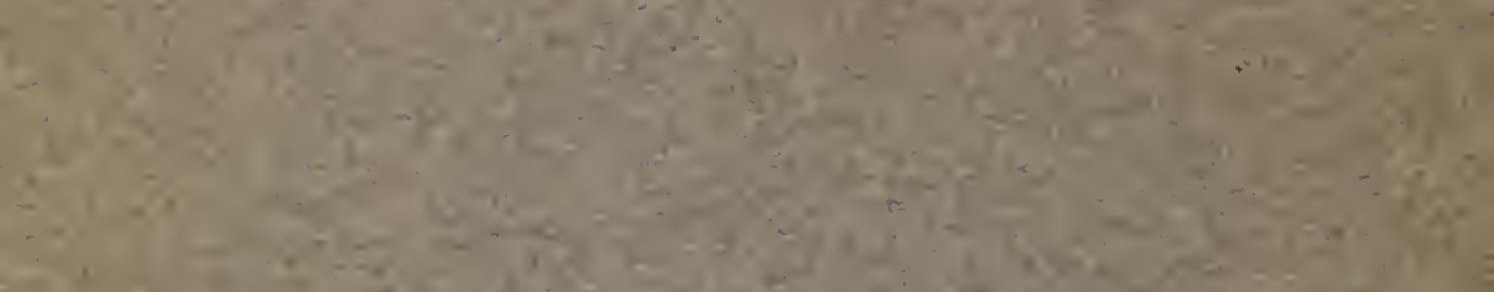

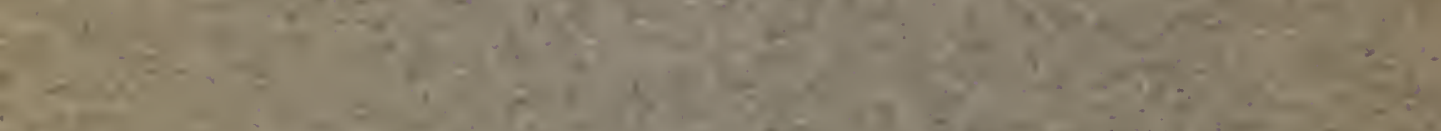
Sisting

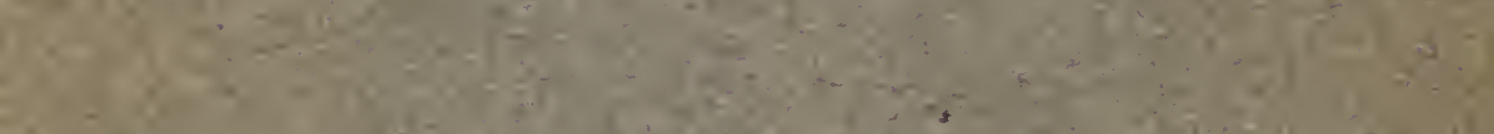

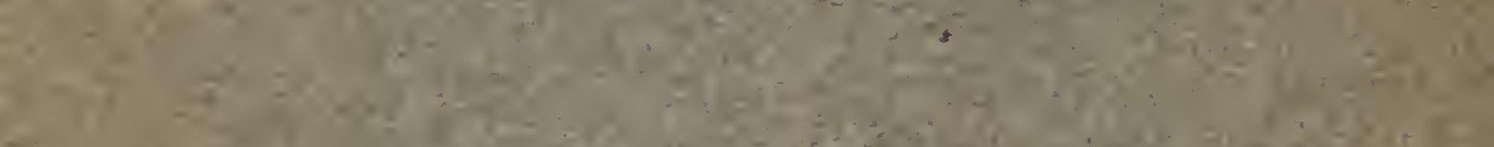

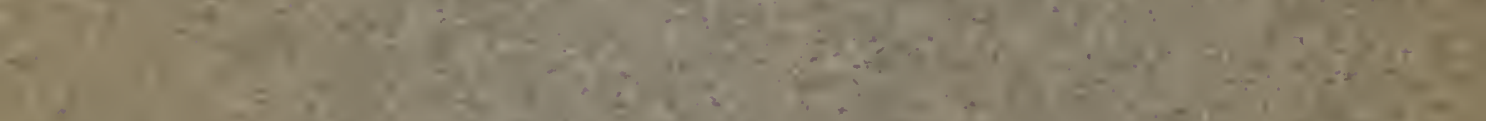

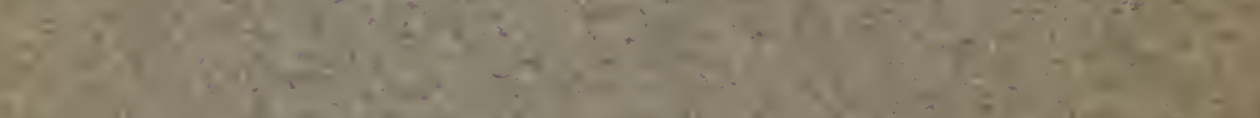

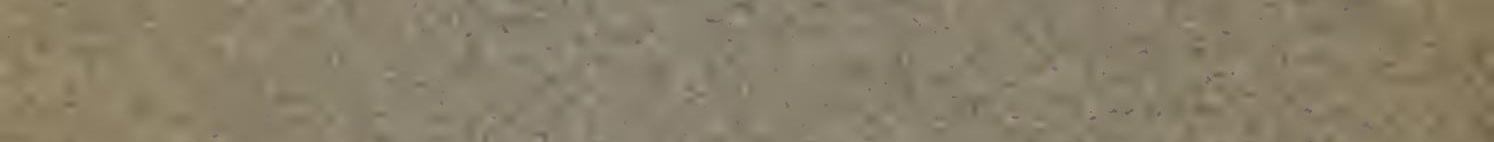

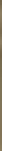

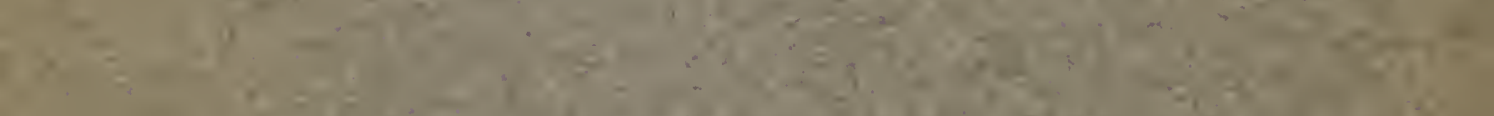

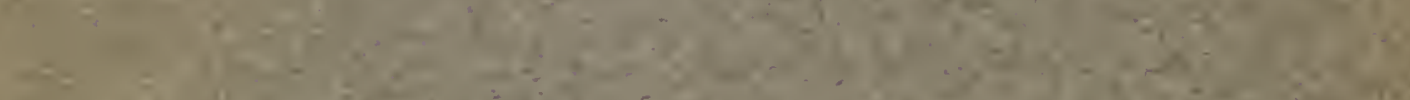

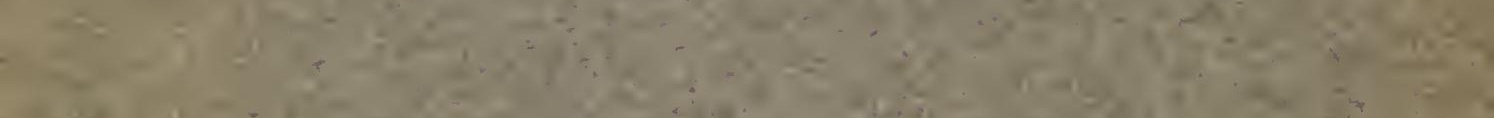

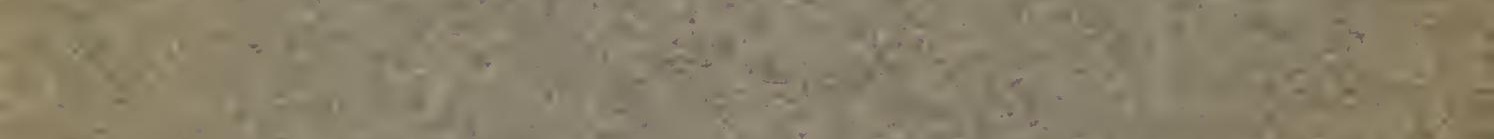

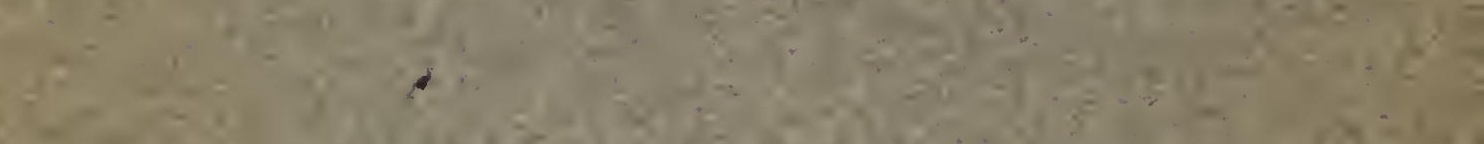

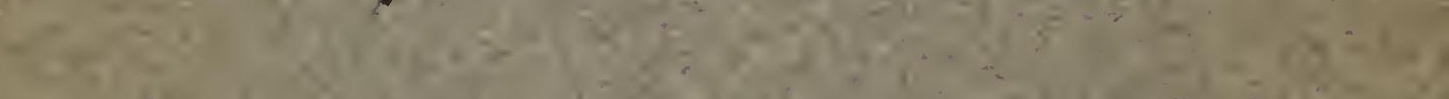
axis

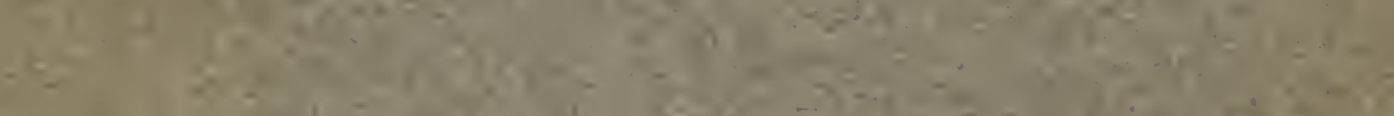

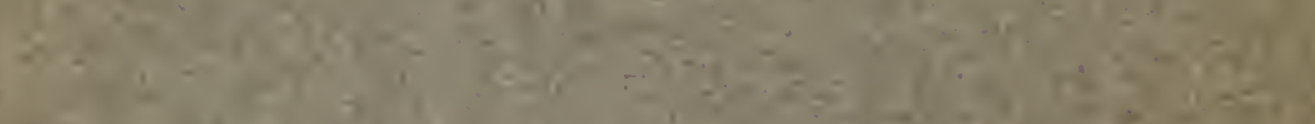

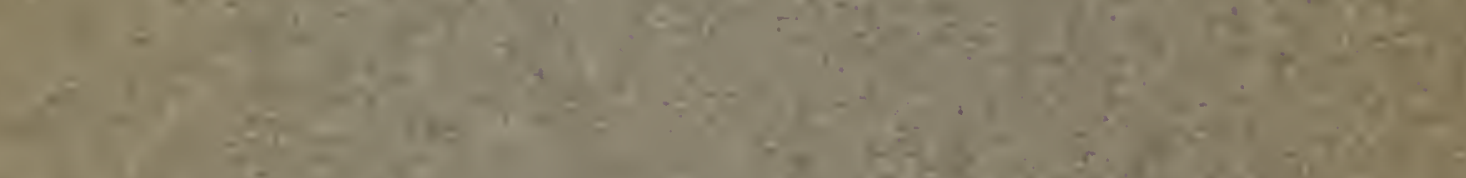

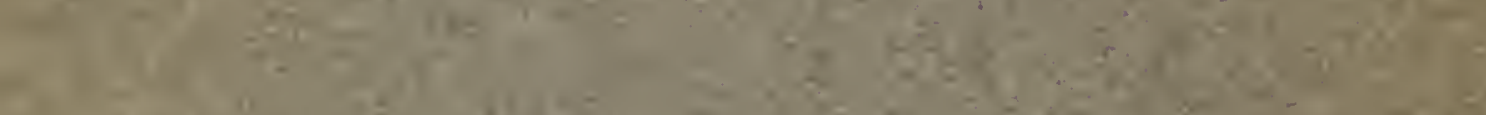

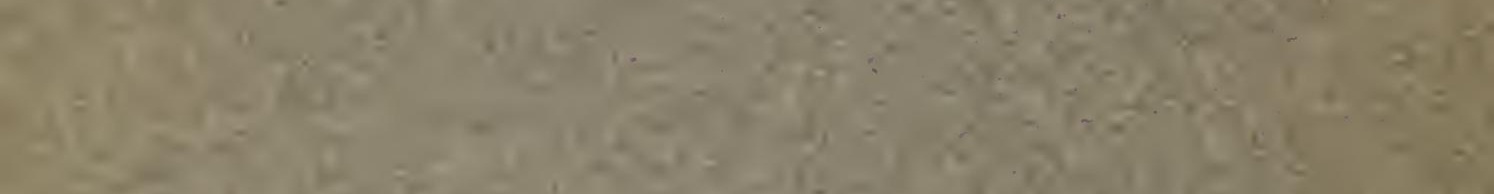

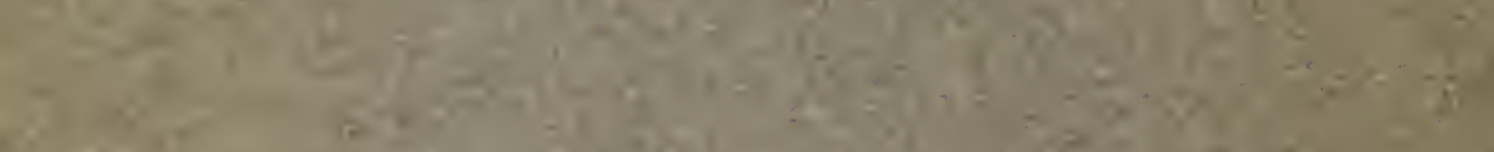

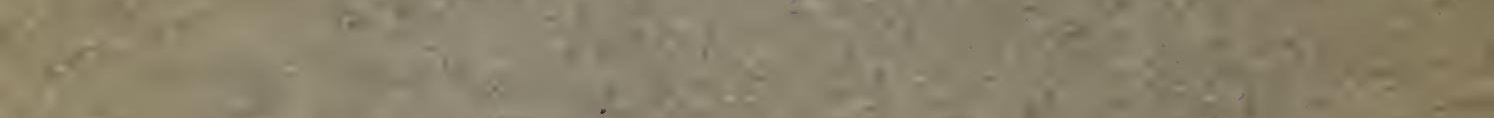

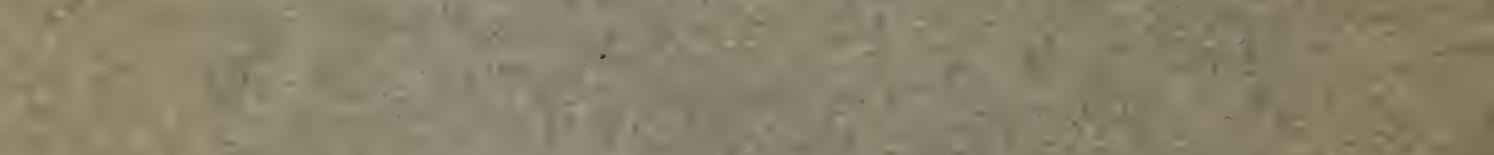

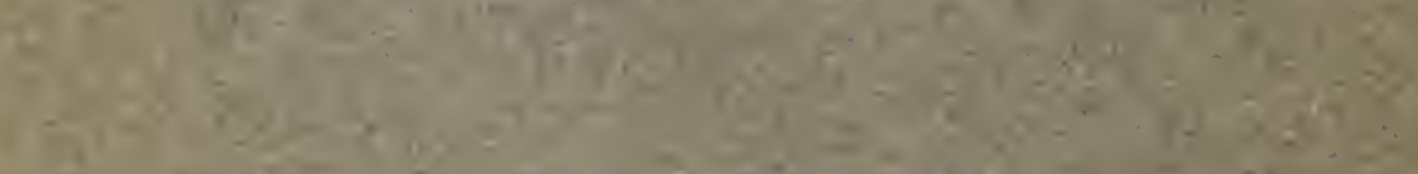

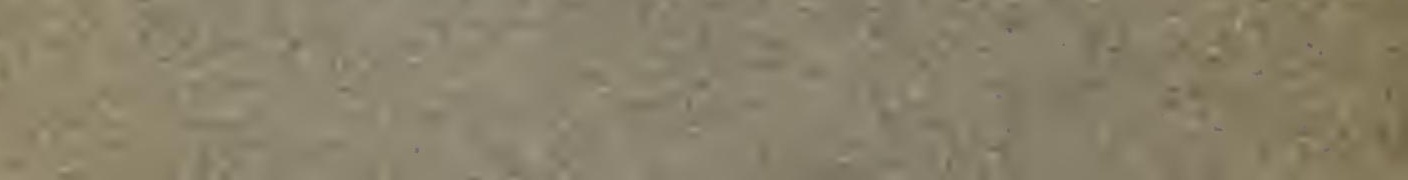




\section{A LIST OF PLAN'TS}

\section{COLLECTED IN BERMUDA IN 1905}

BY

ALBERT HANFORD MOORE

Including a Description of Two New Species

CAMBRIDGE, MASSACHUSETTS

Issued March 12, 1906 



\section{INTRODUCTORY NOTE.}

The following list of plants is the result of collections made by the author in the British Colony of Bermuda in July and the first week of August, 1905.

The peculiar interest presented by the flora of the islands composing this eolony lies in the fact that there is but a eomparatively limited number of endemie species, and that the flora is consequently made up almost entirely of plants derived from elsewhere, either by the agency of man or without it; mainly from the West Indies, but also from North and South Amcrica and even from Asia and Afriea. The plants from the last two eontinents are, however, of the most transient eharaeter, and probably in all cases escapes from cultivation. Zantedeschia æthiopica (L.) Spreng., thongh only three or four specimens were observed, had, curiously enough, made itself quite at home in a marsh comparatively remote from habitation, and was fruiting freely. As in other sueh regions, every stage in the naturalization of plants is to be noted, from the merest garden escapes such as Zebrina pendula Schnitzl and Tropeolum majus $L$., never growing far from a garden site, to such well established plunts as Conocarpus ereetus $L$. and Coffea arabica $L$. The former of these grows everywhere among the rocks of the seashore, while the latter oceupies a limited tract in the Walsingham Distriet, in a deep bowl-shaped depression where are the entrances to the remarkable Walsingham Caves. Here it forms the principal vegetation, and eovers the ground with its seedlings to the almost complete exelusion of herbaceous vegetation. That such plants as these are not native eould only be known from the records. Intermediate between these cases are such plants as the Zantedeschia, above mentioned, which, although searce and obviously introduced, yet seem to be at least temporarily established. Then come the weeds, whieh are rampant everywhere along the roadsides and in the gardens. Among the most conspicuous of these are Ammi majus L., Foniculum vulgare Mill., and Erigeron linifolius Willd. Finally, there are truly native plants such as Asplenium muticmm Gilb., Typha domingensis Pers., Piaropus crassipes (Mart.) Raf., Euphorbia buxifolia Lam., Proserpinaca palustris $L$., and the like. 
The question what plants are native and what are not is fully discussed by Sir W. Botting Hemsley, A. L. S., * and need therefore not be entered into here.

Besides two new species, the additions to Hemsley's list are mostly introduced weeds or garden escapes, and therefore of very trivial importance. But since in the earlicr lists a considerable number of species are recorded on the basis of reports - some of them many years old - or of scanty material, and, especially, since the flora of Bermuda is inadequately represented in American herbaria, it has seemed best to put on record all the species collected.

The specimens upon which this list has been based have been determined (except in a very few cases otherwise indicated) by comparison with specimens at the Gray Herbarium of Harvard University, Cambridge, Massachusetts. The names have been made to accord with the rules of nomenclature adopted by the International Congress at Vienna in July, 1905. The author wishes to express his appreciation of the courtesy cxtended to him by the staff of the Gray Herbarium and its librarian in the use of its specimens and library in the preparation of this pamphlet.

The numbers following the names in the list and enclosed in brackets are the author's collection numbers, the complete set being in his herbarium. An asterisk precedes those names which are not recorded from Bermuda in Hemsley's list. In instances where the first name cited is not the one given in that list, the abbreviation "H. L." follows the name which is there used. This abbreviation is also employed in other cases in which the same work is referred to. The symonymy does not aim at completeness, but is in the main a selection of such synonyms as seemed to be significant in connection with the nomenclature; full citations are given only when it has been found necessary to displace the name in current use, which has not often been the ease, and in two other instances in which it seemed desirable to give them; no new combinations were required.

The pamphlet contains three reproductions from original photographs.

Cambridge, Massalchusetts. 1906.

ALBERT HANFORD MOORE.

* In a list contained in the Report on the Scientific Results of the Voyage of II. M. S. Challenger during the years $1873-76 \ldots$ Botany, Vot, $i$. under the title Report Oceans.
Ony of the Bermudos and vorious other Islands of the Atlantic and Southern 
LIST.

POLYPODIACEA.

Acrostichum aureum L. [3156]

Adiantua bellun Moore. [3119]

Asplenium dentatum L. [3105]

*Aspleniuar nuticua Gilb. [2981, 3102]

Nephrodium patens (Sw.) Desv. [2016, 2017]

Polypodium patens Sw.

Aspidium patens $\mathrm{Sw}$.

Nephrodium villosuin (L.) Presl. [2993]

Polypodium villosum $\mathrm{L}$.

Aspidium villosum (L.) Sw.

Nephrolepis exaltata (L.) Schott. [2979]

Polypodium exaltatum I.

Aspidium exaltatum (L.) Sw.

Polytodiun elasticua Rieh. [3118a, 3129]

Pteris aquilina L. var. caudata (L.) Hook. [2020]

Pteris candata L.

Pteridium caudatum (L.) Max.

*Pteris longifolia L. [2991]

Woodwardia virginica (L.) Sm. [2018, 2998]

Blechnum virginicum $\mathrm{L}$.

Doodia virginica (L.) Presl.

Anchistea virginica (L.) Presl.

OSIIUNDACEA.

Osaunda REgalis L. [2019]

PINACEA.

JUNIPERUS berMUdiana L., with larger fruit, leaves, and branehlets than its near relative $J$. barbadense $L$. in the other West Indies. [2846, 3068] 
TYPHACEA.

Typha domngensis Pers. Sym. Pl. ii, 532 (1807). [2970, 2971] T. angustifolia L. $\beta$. domingensis (Pers.) Griseb. Fl. Brit. IV. Ind. Ist. 1512 (1S64) (Pers. according to H. L.).

PO'TAMOGE'TONACEE.

RUPPIA maritima L. [2\$47]

GRAMINEA.

*avena sativa L. [2953]

*Cenchirus echinatus I. [2951]

Cenchios tribulomes I. [3073]

*Cinftochloa vinidis (L.) Scribn. U. S. Dept. Agr., Div. Agrost. Bull. iv, 39 (1897). [2856, 2S8S, 3040]

Panicum viride L. Sp. Pl. ed. II, 83 (1762).

Setaria viridis (L.) Beauv. Essai d'une Nouv. Agrost. ou Nouv. Genres Gram. 53 (1812).

Ixophorus viridis (L.) Nash Bull. Torr. Bot. Club xxii, 423 (1595).

Chamaraphis viridis (L.) Port. ibid.

Cingris petrasa Sw. (Thunb. according to H. L.). [2912]

Crnodon Dactilon (L.) Pers. [2913, 2936]

Panirum Dactylon L.

Capriola Dartylon. (I.) O. Kitze.

*Eleusine inidica (I..) Gicrtn. [2873]

Cynosurus indicus $\mathrm{I}$.

*Elymes virginicus I. [30t1]

Oplisimens undulatifolits (Ard.) Beauv, Essai d'une Nouv.

Agrost. ou Nouv. Genres Gram. 53 (1812). [3089, 026]

Panicuhum undulatifolium Ard. Veron. Hort. Publ. Pat.

Cust. Aninadv. Bot. Spee. Alter. (1764) (Paniculum err. typ. for Panicum).

P'micum setarinm Lam. Illustr. Genres i, 170 (1791).

Oplismemus setarins (Lam.) R. and S. Syst. Veg. ii, 481 (1817) (H. L.). 
Orthopogon setarius (Lam.) Spreng. L.-Spreng. Syst.

Veg. i, 306 (1825).

Orthopogon undulatifolius (Ard.) Spreng. 1. c.

* Panicum Colonuar L. [3174]

*Panicuni Crus-Galli L. [3001]

Panicum naxinum Jacq. [2850, 3136]

*Panicum sanguinale L. [2906, 2977]

*Paspalum conjugatum Berg. [3126]

Panichm conjugatum (Berg) Roxb.

Paspalum distichum L. [3166]

Some of the Panica and Paspali in H. L. may be misdetermin-

ations on the part of local botanists for some of the above.

*Phleual pratense L. [2958, 3040]

Polypogon aionspeliensis (L.) Desf. [2915]

Alopecurnes monspeliensis $\mathrm{I}$.

*Spartina cinosuroines (L.) Willd. [3175]

Dactylis cynosuroides L.

Sporobolus indicus (L.) R. Br. [2S79]

Agrostis indica L.

Vilfa indica (L.) Trin.

Stenotaphrum diniditum (L.) Brogn. in Duperry Voy. autour du Monde de la Coq. ii, 127 (1829). [2868]

Panicum dimidiatum L. Sp. Pl. 57 (1753).

Rottbolle dimidiata (L.) Lam. Illustr. Genres i, 205 (1791).

Stenotaphrum glabrum Trin. Fund. Agrost. 176 (1820).

This is the Stenotaphrum americanum Schrk. of H. L.

\section{CYPERACEA.}

Cladium effusum (Sw.) Torr. [3157]

Schcenus effusus Sw.

Cladium Mariscus R. Br., which is reported in H. L., is probably this specics. No. 3005 is apparently a too immature though habitally finely developed specimen. Especially in respeet to its achenes it looks a little different, but probably is not.

*Cyperus brunneus Sw. [3078]

*Cyperus esculentus L. [3120]

Dichromena rolorata (L.) A. S. Hitch, 4th Rept. Mo. Bot. Gard. 141 (1S93). [3009] 
Schomus coloratus L. Sp. Pl. 43 (1753).

Dicheomena lencocephala Michx. Fl. Bor. Am. i, 37 (1803) (H. L.).

Eleocharis capitata (L.) R. Br. [3164]

Scirpus eapitatus L.

*Eleocharis interstincta (Valil) R. and S. Syst. Veg. ii, 149 (1817). [3010]

Scirpus interstinctus Vahl Enum. Pl. ii, 251 (1806).

Scirpus equisetoides Ell. Bot. S. C. and Ga. i, 78 (1816).

Eleocharis equisetoides (Ell.) Torr. Ann. Lyc. N. Y. iii, 296 (1S36).

*Fimiristylis spadicea (L.) Vahl. [2969]

Scirpus spadicens L.

Undoubtedly this is a native species.

*Rinyciospori dommucensis A. H. Moore spec. nov. perennis, erecta, herbacea, dense caspitosa, colore alboviridis; eaule terete, canaliculato, cavo; foliis $2-3.5 \mathrm{~mm}$. latis, $1.8-3 \mathrm{dm}$. longis cireum plantre basim persistentibus: inflorescentia perianthi segmentis capillaribus non setaceis: fructus capitibus $5 \mathrm{~mm}$.$2 \mathrm{~cm}$. diametro, bracteis margine perminute ciliatis, squamis fertilibus ovatis, nervo prominente, apice mucronato, flavofuseis nondum leviter rufescentibus, squamis vacuis flavis, lanceolatis acuminatis: achenia lenticularia, glabra levia nitida, flavofusca vel leviter rubescentia, minime colore e nigro rutilo, præter rostrum ca. $2 \mathrm{~mm}$. longis, 1.6-1.8 mm. latis; rostro latitudine apucl basim achænio requali, .2-.5 $\mathrm{mm}$. longo, apice subobtuso. In subsalsa palude cui nomen dommucensis crescit.

R. glomerata (I..) Vahl speciei nostrae simillima ab eadem facile distinguitur achæeniis .9-1.1 mm. longis, rostro .6-1 mm. longo, peracuminato.

R. capillacea Torr. caulibus foliisque percapillaceis.

R. cymosa Ell. et R. alba (L.) Tahl acheniis percorrugatis.

R. fascicularis (Mich.x.) Vahl et R. distans (Michx.) Vahl achaniis colore e nigro rutilo, medio luteis.

Nomen a parochia dommucensi (Devonshire) inditum. De hac specie, ut videtur, v. cl. W. Botting Hemsley 1. c. scripsit "Rhynehospora sp. n."

Specimen typicum sub numero 3004 in Herbario A. H. Moore depositum est.

Videsis tabulas I et II infra. 
Rhynchospora stipitata Chapm. [3007]

Scirpus validus Vahl. The S. lacustris L. of H. L. [3006]

\section{PALME.}

*Sabal Adansoni Guems. [3142]

Corypha minor Murr.

Sabal minus (Murr.) Pers.

ARACEN.

*Zantedeschia zthiopica (L.) Spreng. L. - Spreng. Syst. Veg. iii, 765 (1S26). [2966]

Calla athiopica L. Sp. Pl. 968 (1753).

Colocasia athiopica (L.) Lk. Diss. Bot. Suerin, 77 (1795).

(For eitation see Hook. f. and Jaeks. Ind. Kew.)

Richardia africana Kunth in Mém. Mus. Par. iv, 433 (181S).

(For citation see Hook. f. and Jacks. 1. c.)

Richardia athiopica (L.) Spreng. L. - Spreng. l. c.

LEMNACE $A$.

LEMNA MINOR L. [3021]

\section{COMNLLINACEA.}

Commelina nudiflora L. [2880]

*Zebrina pendula Schnitzl. [2015, 2945]

\section{PONTEDERIACEA.}

* Piaropus crassipes (Mart.) Raf. Nov. Tell. S1 (1S36). [3000] Pontederia crassipes Mart. Nov. Gen. et Sp. i, 9 (1824). Eichornia speciosa Kunth Enum. Pl. iv, 131 (1843).

Eichornia crassipes (Mart.) Solms ex A. and C. DC. Mon. Phan. iv, 527 (1883).

JUNCACEA.

Juncus ACUTUS T. [2892]

Juncus marginatus Rostl. [300S] 


\section{LILIACE 2 .}

*Lilium longiflorua Thunb. [3138]

Lilium candidum Thunb.

Lilium Harrisii Carr.

Lilium eximium Court. and

Litium longiflorum Thunb. var. eximium (Court.) Nichol.

(The cultivated kind known as Easter or Bermuda Lily.)

YuCCA ALOIFOLIA L. [3049]

YUCCA GLORIOSA L. [3139]

\section{AMARYLLIDACEE.}

Agave americana L. [3141]

*Hymenocallis caribea (L.) Herb. [3147]

Pancratium caribaum L.

*Pancratiuli marithium L. [2932]

IRID ACEA.

Sisyrinchium Beriudinnum L. [2919, 298S]

MARANTACEL.

*Miranta arundinacea L. [3135]

PIPERACEE.

Peperoma magnoliferolia (Jacq.) A. Dietr. [3107]

Piper magnoliafolia Jacq.

\section{MYRICACEAE.}

Myrica cemifera $L$. This is the real Myrica cerifera $L$. as distinguished from Myrica carolinensis ifill. [297S, 3151]

\section{UIMACE.E.}

Celtis occinentalis I. [3109]

Celtis pumila Pursh.

Celtis crassifolia Lam. 
Trema Lamarchiana (R. and S.) BI. Mus. Bot. Lugd.-Bat. ii, 59 (1852-1856). [3110]

Celtis Lamarclizana R. and S. Syst. Veg. vi, 311 (1820)

Sponia Lamarckiana (R. and S.) Decsne. Nouv. Ann. Mus. Par. iii, 498 (1834) (H. L.).

\section{URTICACEE.}

Behmeria crimndrica (L.) Sw. Prod. Veg. Ind. Occ. 234 (1788) (Willd. according to H. L.). [2997]

Urtica cylindrica L. Sp. Pl. 1396 (1753).

Parietaria debilis Forst. f. (Forst. according to H. L.). [3128]

Pilea aitcrophylla (L.) Liebm. [3056]

Parietaria microphylla L.

Urtica microphylla (L.) Sw.

Pilea muscosa Lindl.

Urtica URens L. [2959]

\section{POLYGONACE无.}

Coccoloba uvifera L. (Jacq. according to H. L.). [3031, 3032, 3079]

Polygonum wiferum L.

RuMEx CRISpes L. [2956]

\section{CHENOPODIACEA:}

Chenoponiun albuit L. [2914, 2935, 2950]

Chenofodum ambrosiomes L. var. anthelmaticun (L.)

Gray. [2934, 2983]

Chenopodium anthelminticum L.

Saimornia fruticosa L. The Salicornia ambigua Michx. of H. L. [2939]

\section{AMARAN'TACEF.}

Amarantus hybridus L. [2886]

A marantus chlorostachys Willd.

*Ailarantus retroflexus L. [2948] 
BASELLACEE.

Boussingaultia Baselloides HBK. [2929]

NYCTAGINACEA.

Berhavia erecta L. [3106, 3134]

AIZOACEA.

Sesuvium Portulacastrum L. [2933]

PORTULACACEA.

Portulaca oleracea L. [2S72]

PAPAVERACEA.

Argenone mexicana L. [2854, 2855]

Fumaria muralis Sond. ex Koch Sỵn. Flor. Germ. et Hcty. ii, 1017 (1St4). [2920]

Fumaria Petteri Koch l. c. i, 435 (1843), not Reichenb. The Fumaria caprcolata $L$. of H. L.

Papaver somniferun L. [2955]

\section{CRUCIFERA.}

*Brassica nigra (L.) Koch. [2927, 2957]

Sinapis nigra L.

Cakile dequalis L'Her. (L. according to H. L.). [2897, 2904, 2905, 2911]

Coroxopes minuts (L.) Sm. Fl. Brit. iii, 691 (1800). [2S63]

Lepidium didymum I. Mant. 92 (1767).

Sencbiera didyma (L.) Pers. Syn. Pl. ii, 185 (1S07).

Senebiera pimuatifida D)C. in Mém. Soc. Hist. Nat. Par. 14t, t. \& (1799) (H. L.).

Lepidum virginicum I. [2852]

Mattilola incana (L.) R. Br. [3046, 3077]

Cheiranthus ineanus. $\mathrm{L}$.

Raphanes Raphanistrua L。 [3014] 
*Raphanus sativus L. [28S4] Sistumrium officinale (L.) Scop. [2857]

Erysimum officinale L.

\section{CRASSULACEÆ.}

Bryophylum pinnatum (Lam.) Kurz in Journ. As. Soc. Beng. $x 1,2,52$ (1S71). (For citation see Hook. f. and Jacks. Ind. Kew.) [2908, 3036]

Cotyledon pinnatum Lam. Encycl. Meth. (Bot.) ii, 141 (1786). Bryophyllum calycinum Salisb. Parad. Lond. t. 3 (1805) (H. L.).

\section{LEGUMINOSE.}

Canavali obtusifolia DC. (Canavalia in H. L.). The genus was first published by Adanson as Canavali, a native East Indian name of feminine gender. [3081]

*Indigofera Anil L. [3125]

LeuCena GlauCA (L.) Benth. [2961, 2962, 3133]

Mimosa glauca L.

Acacia glauca (L.) Mœnch.

Melilotus officinalis (L.) Lam. [2890, 2910]

Trifolium M. officinalis L.

*Trifoliuir hýriduir L. [2941]

*Trifolium pratense L. [2940]

*Trifolium repens L. [3144]

Vigna luteola (Jacq.) Benth. [3127]

Dolichos luteolus Jacq.

OXALIDACE无.

Oxalis corniculata L. [2921, 024]

TROPEOLACEA.

*Tropeolua uajus L. [2989]

LINACEAE.

Linum usitatissimum L. [2924] 


\section{RUTACEA.}

Citrus Aurantium L. [3091, 3092]

Citrus Linonuli Riss. [3099]

SIMARUBACEE.

SURIANa maritima L. [2952, 30t8]

MELIACEA.

Melia Azederach L. [2930, 2931]

\section{EUPHORBIACEA.}

Croton maritiuus Walt. [3080]

Euphoribia buxifolia Lam. [2902]

Chamasyce buxifolia (Lam.) Small.

Euphorbia heterophylla L. [3087, 027]

Poinsettia heterophylla (L.) Small.

*Euphorbia heterophylla L. var. graminifolia Engelm. [3039]

Euphorbia maculata L. var. Detonsa Engelm. ex descr. in

DC. Prod. This is doubtless the E. maculata $L$. of H. L. $[2870,3074,3123]$

Euphorbia nutans Lag. Gen. et Sp. 17 (1816). [2875, 2974]

Euphorbia Prestii Guss. Fl. Sic. Prod. i, 539 (1827). (For citation see Mem. Torr. Bot. Club v, Sig. 14, 214.) This is the Euphorbia hypericifolia $L$. of H. L.

Euphorbia Peplus L. [2869, 2922, 3167]

Tithymahs Pephus (L.) Hill.

Euphorbia prostrata Ait. [2871]

Chamasyce prostrata (Ait.) Sinall.

Mercurialis annua L. [2858, 2972]

Phyllanthus Niruri I. [2973]

Ricinus comiunis L. [3082, 3137]

ANACARDIACEE.

Ruus Toxicodendron I. [3037] 


\section{AQUIFOLIACEAE.}

Ilex vomitoria Ait. Hort. Kew. i, 170 (1789). [2895]

Ilex Cassine Walt. Fl. Car. 241 (1788), not L. This is the Ilex Cassine $L$. of H. L.

\section{CELASTRACEA.}

*Elemodendron Laneanum A. H. Moore spee. nov., arbor 12-18 $\mathrm{m}$. alta, plerumque ad 2-3 $\mathrm{m}$. a terra eopiose ramifieans; foliis obovatis, eonspieue mueronulatoerenatis, margine parum vel omnino non revoluto, apud basim attenuatis, brevipetiolatis, glabris non nitidis, 4.5-7 em. longis, 2.5-3.5 em. latis; inflorescentia ex eorymbis densis umbelliformibus eonstante, floribus brevipeduneulatis (peduneulis .1-1.4 em. longis, pediecllis $3-5 \mathrm{~mm}$. longis), nee dichotome nee triehotome ramifieante; fruetibus plerumque bini, brevipedunculatis, apiee mueronulato sed raro vel nunquam attenuato; in regione walsinghamensi in paroehia hamiltonensi in siceo ereseens.

E. xylocarpum (Vent.) DC. (Cassine xylocarpa Vent.) a nostra specie longe differt foliis fere integris, vix vel nullo modo apud basim attenuatis ; fruetu obtuso.

E. attenuatum Rich. fruetu longe attenuato; foliis nitidis, fere integris margine revolutis.

E. dioieum Griseb. foliis maioribus (8.5-12.5 em. longis, 4.5$9.5 \mathrm{em}$. latis) apud basin vix attenuatis.

Tres speeies illæ a nostra longe distant infloreseentia diehotome vel trichotome ramifieante.

Specimen florens Elæodendri Laneani A. H. Moore in Herbario Grayano titulum fert "M. Lane no. 365. Bermuda": nisi fallor v. el. A. W. Lane existimavit hanc novam varietatem esse, nam in eodem titnlo nomini Elæodendri xylocarpi (Vent.) DC. appellatio varietatis inedita subiuneta est. Cuius rei memoria novan speeiem E. Laneanum nominavi. V. el. W. Botting Hemsley 1. e. seripsit "Elæodendron xylocarpum, DC. . . . Speeimina bermudiana ab is India oecidentalis diffcrunt foliis angustioribus, deorsum attenuatis, dentibus distinete apieulatis instructis; floribus etiam paullo majoribus - fruetus deest... The Bermudan plant should perhaps rank as a distinet species, but the speeimens are insufficient to settle this point." 
Specimen typicum in Herbario A. H. Moore sub numero 3111 depositum est.

Videsis tabulam III infra.

\section{SAPINDACE E.}

Cardiospermum Halicacabum L. [3088, 3098]

Cardiospermum microcarpum HBK. (both H. L.).

Dodonze viscosa Jacq. The variety angustifolia $L$. $f$. reported in H. L. does not seem to be clearly separable from the foregoing. [2861]

TILIACEA.

Triumfetta semitriloba Jacq. [3097]

MALVACEE.

Malya parviflora L. [3093]

Sida carpinifolia L. [2883, 3095]

GUTTIFERA.

Ascyruit hypericoides L. Sp. Pl. 788 (1753). [2996]

Ascyrum Crux-Andrea L. Sp. Pl. ccl. II, 1107 (1763).

TAMARICACEE.

Tamatix gallica L. [2903, 2995]

PASSIFLORACEA:

*Passiflora minima L. [3060]

l'assiflora suberosa L.

CARICACEX.

*Carica Papaya L. [3053, 3054] 
CACTACEA.

*Cereus compressus Mill. [3051]

Cereus triangularis Haw.

Opuntia tuna Mill. [2893, 2928, 2990, 3022]

LYTHRACEAE.

Ludwigia Repens Sw. [3011]

PUNICACEAE.

Punica Granatum L. [3066, 3086]

RHIZOPHORACEAE.

Rhizophora Mangle L. [293S, 3154, 3155]

COMBRETACEAE.

Conocarpus erectus L. (Jacq. according to H. L.). [2864, 2900]

ONAGRACE正.

Enothera rosea Soland. (Ait. according to H. L.). [2926] CEnotneiza sinuata L. var. hinsuta T. and G. [2954]

Enothera mexicana Spach. This is the CEnothera sinuata L. of H. L.

HALORRHAGIDACE E.

*Proserpinaca palustris L. [3012]

UMBELLIFERT.

Ammi Majus L. [28S9, 2964] 
Apium graveolens L. [2963, 3148]

Centella asiatica (L.) Urb. in Mart. Fl. Bras. xi, 1,287 (1879). [3002]

Hydrocotyle asiatica L. Sp. Pl. 234 (1753).

Feniculum vUlgare Mill. (Gærtn. according to H. L.). [2891] Anethum Fceniculum L.

Foniculum Fceniculum (I.) Karst.

Torilis nodosa (L.) Gertn. Fruct. et Sem. i, 82 (1788). [2878] Tordylium nodosum L. Sp. Pl. 240 (1753).

Caucalis nodosa (L.) Scop. Fl. Carn. ed. II, i, 192 (1772) (H. L.).

\section{PRIMULACEÆ.}

Anagallis arvensis L. $[2969,3159]$

\section{OLEACEE.}

Jasminum Calopiyllum Wall. ex DC. Prod. viii, 310 (1844); Wall. Cat. no. 2889 (1828) (nom. nud.). [3059]

Jasminum courtallense Wight Ic. Pl. Ind. Or. t. 1252 (1840) (no description).

Jasminum simplicifolium Forst. f. [3083]

Jasminum gracile Andr. (H. L.).

\section{GENTIANACEÆ.}

Erythixa pulchella (Sw.) Fr. [2849, 023]

Gentiana pulchella Sw.

Gentiana ramosissima Vill.

Erythroa ramosissima (Vill.) Pers. (H. I..). This probably also includes the Erythrea texensis Griseb. and Erythræa Centaurium Pers. (H. L.).

\section{APOCYNACEA.}

Nerium Oleander L. [2867, 2896, 2944, 3023, 3030] 
ASCI.EPIADACEA:

Asclepias curassavica L. [3177]

CONVOLVULACEA.

*Evolvulus alsinordes (L.) Willd. [3034]

Iponica Jamaicensis (Spreng.) G. Don. [3058]

Convolvulus jamaicensis Spreng.

Iponatea Pes-Caprea (L.) Sweet. [2898, 3077]

Convolvulus Pes-Capro L.

Iponiea Sagittata Poir. (Cav. according to H. L.). [3171]

IPONGa sInUata Ort. [3130]

Ipomoa disseeta Pursh, not Convolvulus dissectus Jaeq.

HYDROPHYLLACEA.

Nama jamaicense L. [2859]

Conanthus jamaicensis (L.) Hell.

\section{BORRAGINACEA.}

Heliotropium curassavicum L. [2865]

TOURNEFORTIA GNAPHALOdEs (L.) R. Br. [2901]

Heliotropium gnaphalodes L.

VERBENACEF.

Avicennia nitida Jacq. [2937]

*Citharexyluar quadrangulare Jacq. [3085, 3131, 3132]

Duranta Repens L. Sp. Pl. 637 (1753). [2862]

Duranta ereeta L. I. c.

Duranta Plumieri Jacq. Select. Stirp. Am. 186, t. 176, f. 76 (1763) (H. L.).

LANTANA CAMARA L. [2894]

Lantana involucrata L. [2845] 
*Lantana polyacantha Schauer ex char. [3143]

Lippia nodiflora (L.) Michx. [2925]

Verbena nodifora L.

Stachytarpheta Jamaicensis (L.) Vahl. [2866, 021]

Verbena jamaicensis L.

*Verbena bonariensis L. [2946]

*Verbena littoralis HBK. [2939a]

Verbena URTICEFolia L. [287t, 2947]

*Verbena venosa Gill. and Hook. [2984]

\section{LABIAT E.}

Clinopodium Calamintha (L.) O. Ktze. Rev. Gen. Pl. 515 (1891). [2848, 2985]

Melissa Calamintha L. Sp. Pl. 593 (1753).

Calamintha officinalis Mœench. Meth. 409 (1794) (H. L.).

*Coleus scutellarioides (L.) Benth. [3152]

Ocimum scutcllarioides I.

*Leonotis nepeteffolia (L.) R. Br. [3090]

Phlomis nepctafolia L.

Leonurus sibiricus L. [2987]

*Mentha citrata Ehrh. [3170]

*iIentha viridis L. [2999]

Salvia coccinea L. var. pseudo-coccinea (Jacq.) Gray. [3025, $3124]$

Salvia psendo-coccinea Jacq. This is in all probability the Salvia coccinea $L_{\text {. }}$ of II. L.

Salvia serotina L. [2986]

\section{SOLANACEE.}

Datura Stramoniun I. [3053, 3067]

*Lycopersicuar escelentuli Mill. [3168]

Solrumen Lycopersicum L.

Lycopersicum Lycopersicum (L.) Karst.

Nicandra pitsalomess (I.) Gartn. [3084]

Atropa physaloides I.

Physalis peruviana Mill. 
Physalodes peruvianum (Nill.) O. Ktze.

Physalorles Physalodes (L.) Britton. Probably the Physalis peruviana $L$. of $\mathrm{I}$. $\mathrm{L}$. is also to be included.

Nicotiana glauca Grah. [2943]

Nicotiana Tabacum L. [3153]

*Phrsalis angulata L. [2860, 3112]

*Physalis barbadensis Jacq. These specimens are the socalled var. obscura (Michx.) Rydb. (Physalis obscura Michx.),

which does not scem to be very distinct. [3042, 3043]

*Sotanua tuberosum L. [2965]

\section{SCROPHULARIACEA.}

CapraRia biflora L. [3094, 3096]

Monniera calycina (Forsk.) O. Ktze. Rev. Gen. Pl. 463 (1891).

[3013]

Limosella calycina Forsk. Fl. Eg. Arab. 112 (1775).

Ilerpestis Monniera HBK. Nov. Gen. et Sp. ii, 366 (1817) (H. I.).

Monniera Monniera (L.) Britton. Mem. Torr. Bot. Club v, Sig. 19, 292 (August 28, 1894).

*Russelia Juncea Zucc. [3027]

Verbascum Thapsus L. [3028]

ACANTHACEE.

*Dianthera secunda (Vahl) Griseb. [3033]

Justicia secunda Vahl.

PLANTAGINACEE.

Plantago lanceolata L. [2916]

Plantago major L. [2917]

RUBIACEF.

Coffea arabica L. $[3100,3101]$ 
Pstchotria undata Jacq. $[3117,311 \mathrm{~S}]$

Randa aculeata L. [3070]

Relbunium hy pocarpiun (L.) Hemsl. [2637, 3069]

Valantia hypocarpia L.

Rubia hypocarpia (L.) DC.

SPermacoce tenuior L. [29-19]

\section{CAPRIFOLIACEE.}

*Sambucus canadensis L. [3064]

\section{CUCURBITACEE.}

*Citrullus rulgaris Schrad. [3029]

*Cucurbita Pepo L. [3140]

\section{GOODENIACEA.}

Scatola Plumieri (L.) Vahl Symb. Bot. Pl. Arn. ii, 36 (1790). [3072]

Lobelia Plumierii I. Sp. Pl. 929 (1753).

Scarola Lobelia Murr. L.-Murr. Syst. Veg. 17 (177t) (L. according to H. L.).

\section{CONIPOSITAE.}

Ambrosia arteinsiefolia L. [?960] *Anthenis Cotela L. [3055]

Binens pilosa I. [2587, 2992]

Bidens lencantha Willd.

Borricita Ariorescens (L.) DC. [2S51, 2907]

Buphthalmum arborescens $\mathrm{I}$.

*Cirrisanthemula Leucanthenua L. var. subpinnatifiduad Feril. The forms collected were somewhat intermediate between the type and the variety. [2994, 30t4]

Cichoriem Intrbus L. [2SS1]

FCLipta erecta L. [3165] 
Erigeron canadensis I. [2923]

Erigeron Dareilianus Hemsl. [2S76, 020]

Erigeron linifoluts Willd. [2952]

Erigeron philadeliphicus L. [3075]

*Helianthus annuus L. [3071]

Parthenium Hysterophorus L. [2885]

Pluciea Camphorata (L.) DC. $[3162,3173]$

Erigeron camphoratum L.

Conyza camphorata (L.) Ell.

Polminia Uvedalia L. [3061]

Senecto vulgaris L. [3149]

Solmago sempervirens L. [2909, 315S, 025]

Sonchus oleraceus L. [28S2, 2918]

Taraxacum officinale Web. [3062, 3160]

Leontodon Taraxacum $\mathrm{L}$.

Taraxacum Dens-Leonis Desf.

Taraxacum Taraxacum (L.) Karst. 


\section{SPECIES ENDEMIC IN BERMUDA.}

\section{POLYPODIACEA.}

Adiantum bellua Moore. [3119]

Asplenium Laffanianum Bak.

Nephrodium Bernudianum Bak.

\section{PINACEA.}

JUNiPERUS BERMUdiANA L. [2846, 3068]

\section{CIPERACE $A$.}

Carex beruudiana Hemsl.

*Rhynchospora domiteensis A. H. Moore. [3004]

$$
\text { PALAIE. }
$$

Sabal Blackburniana Glazebr.

\section{IRIDACE E.}

Sistrinchium bermudanum L. [2919, 2988]

CELASTRACEA.

*Eideodendron Laneanum A. H. Moore. [3111]

\section{PLUMBAGINACEA.}

Statice I,ferroy Hemsl.

COMPOSITAE.

Eargeron Darelutanus Hemsl. [2876, 020] 
PiATE:

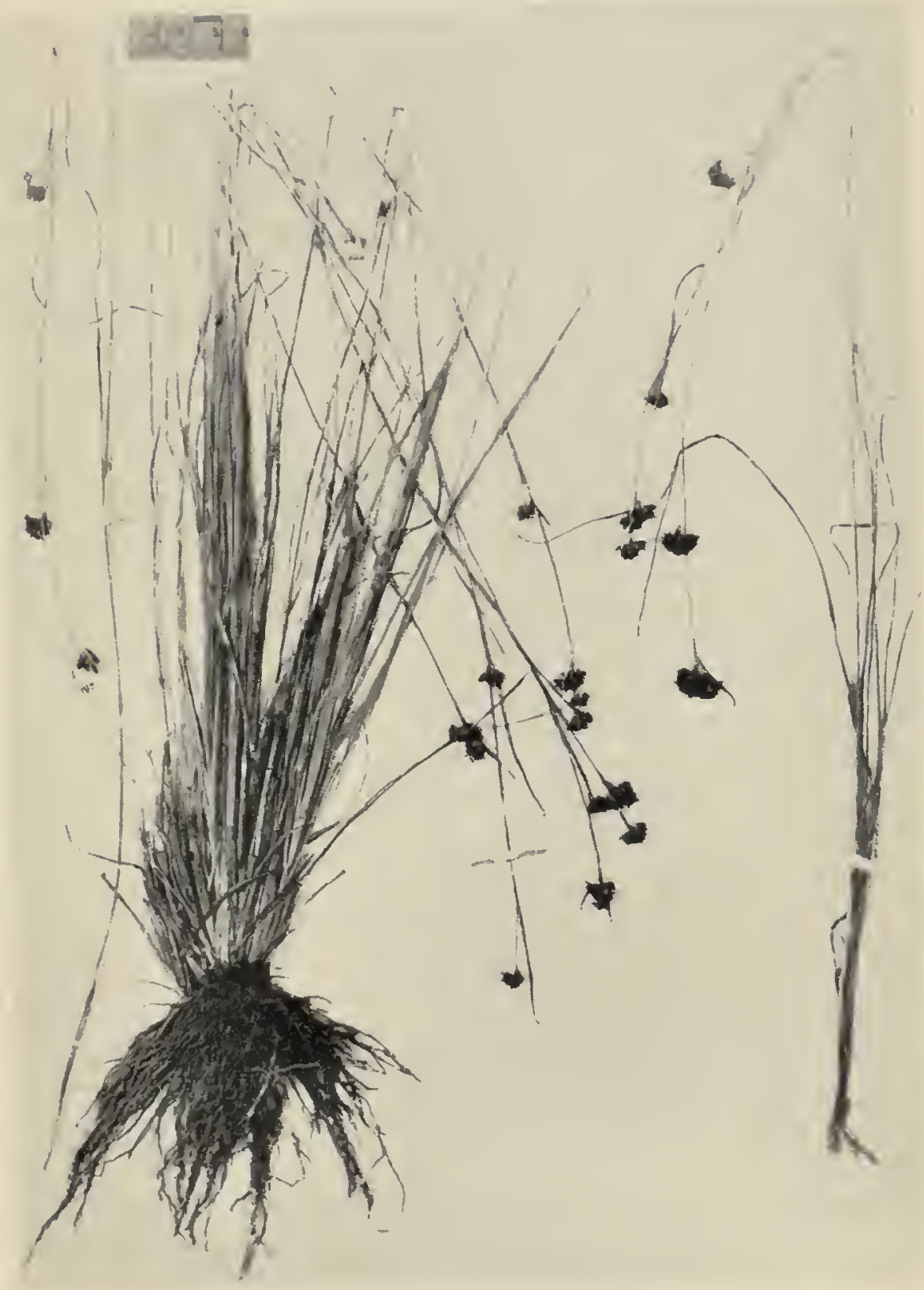

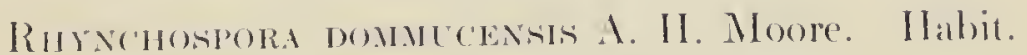



PI.ITE II.

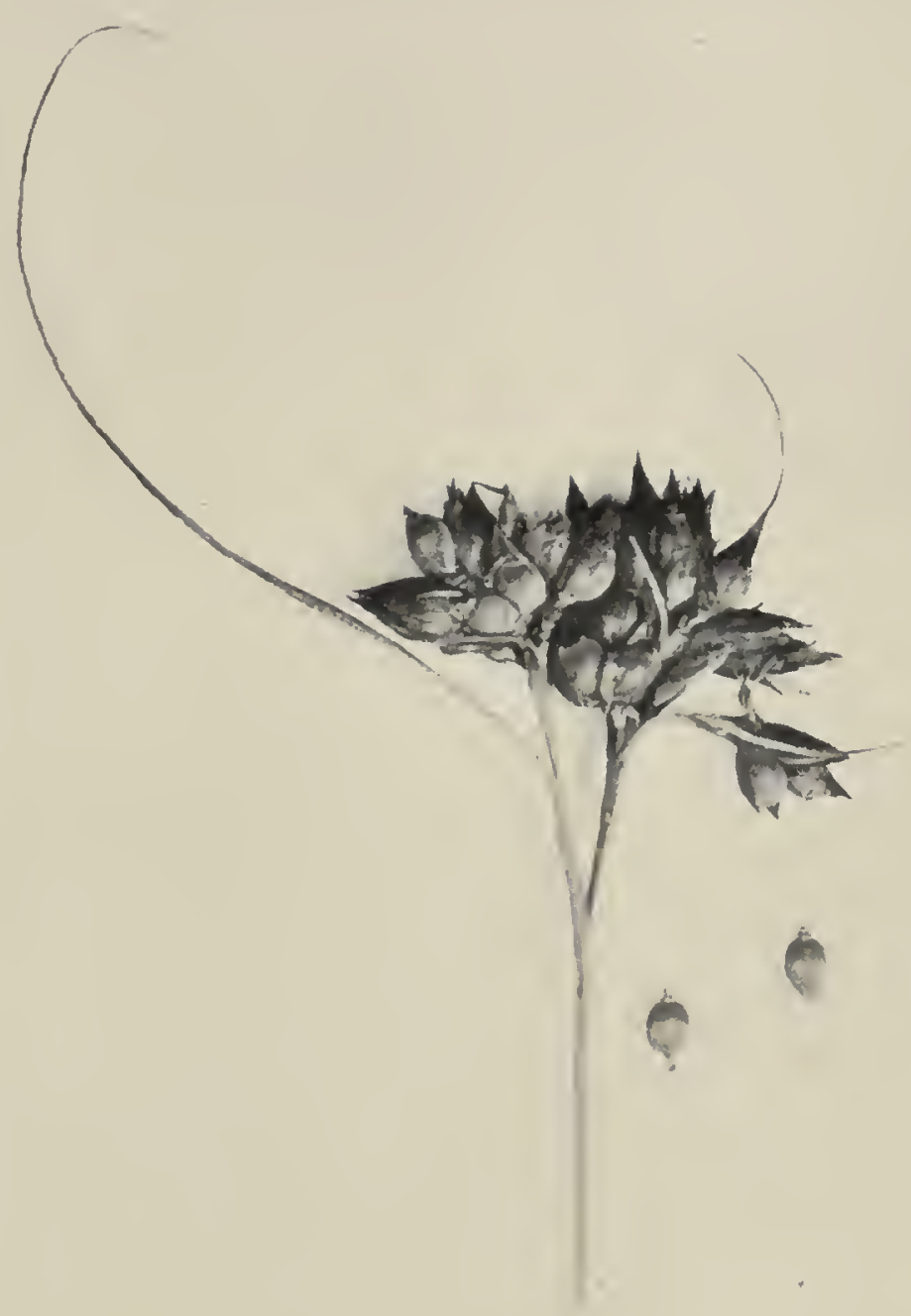

Rhrochospora dommerensis A. H. Moore. Head and achenes, magnificd :3 diameters. 

PLATE III.

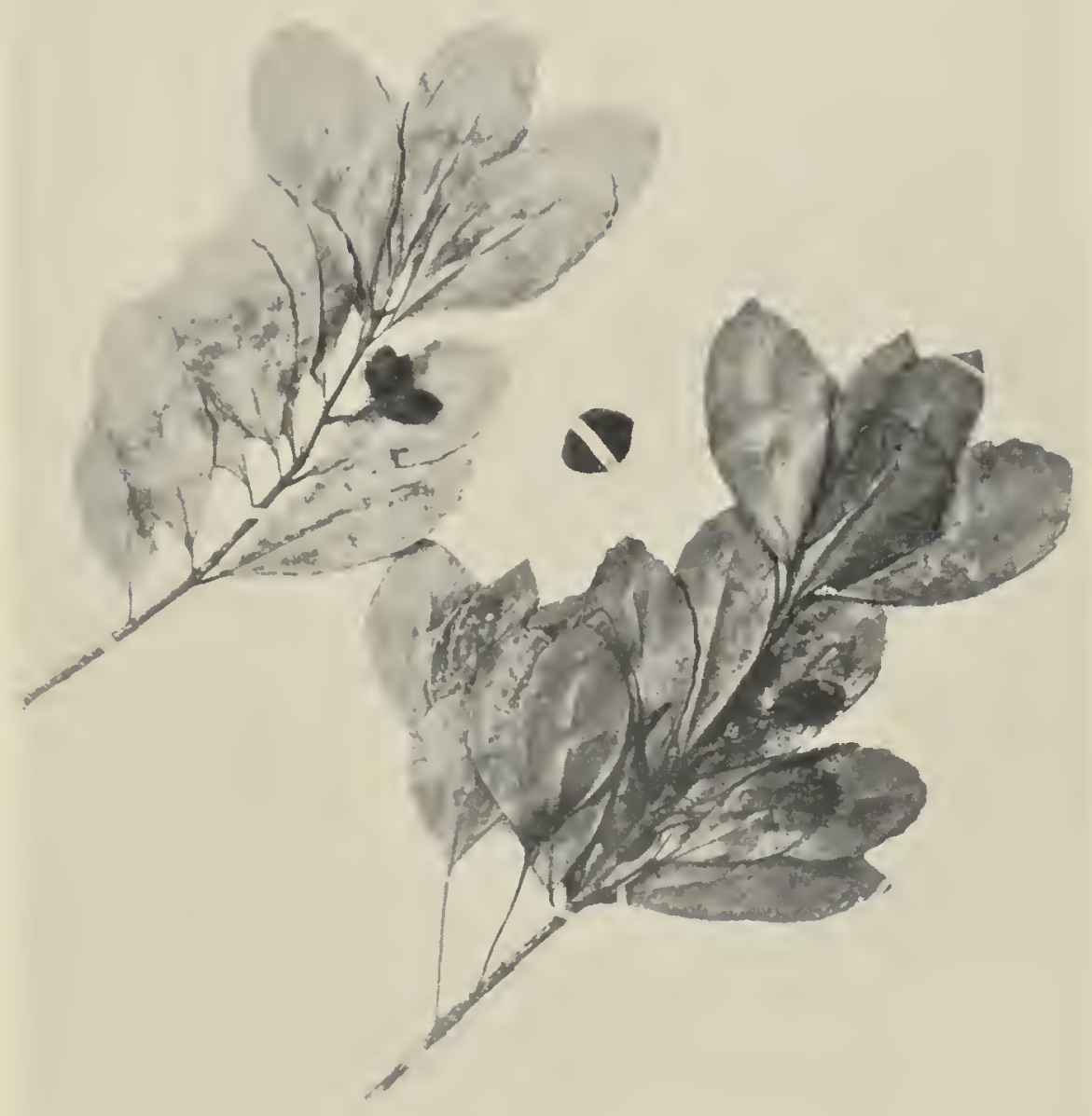

Elloumenon laneanum A. H. Migore. 



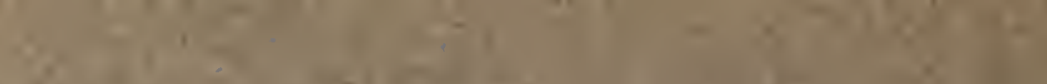

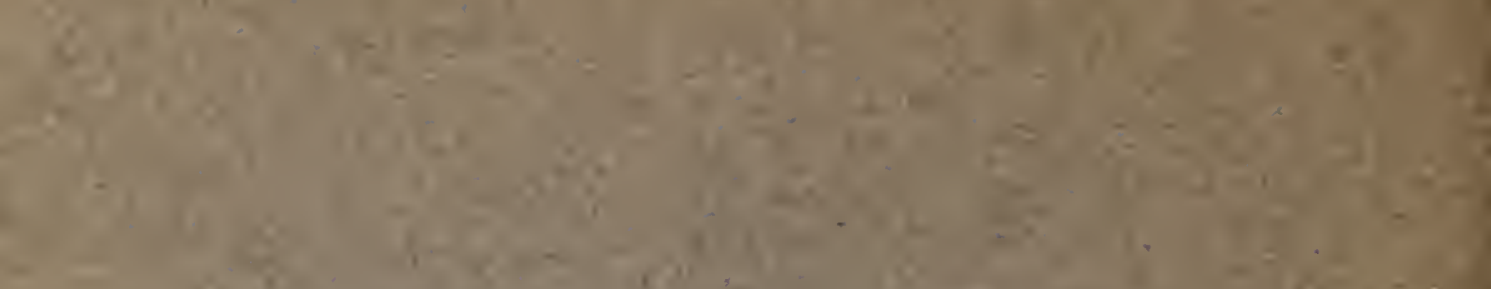
and

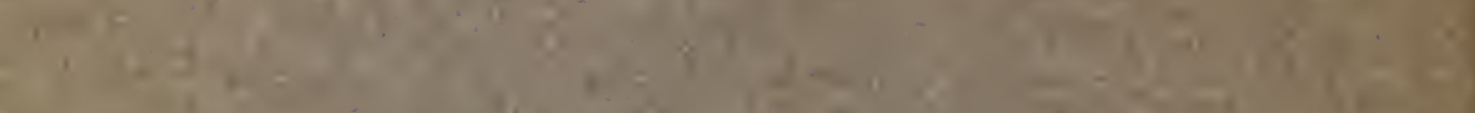

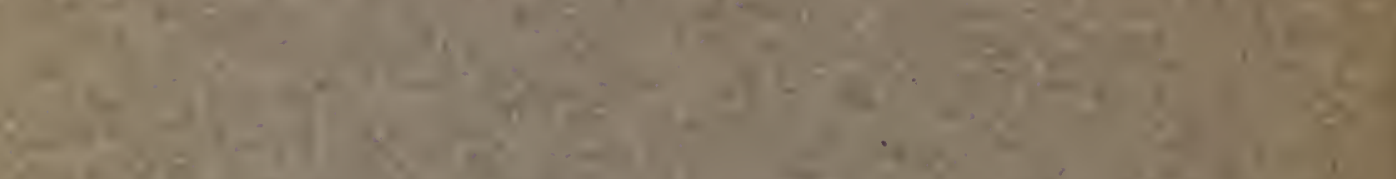

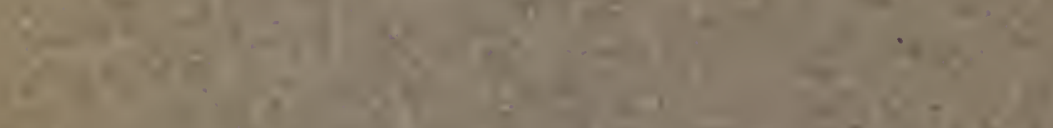

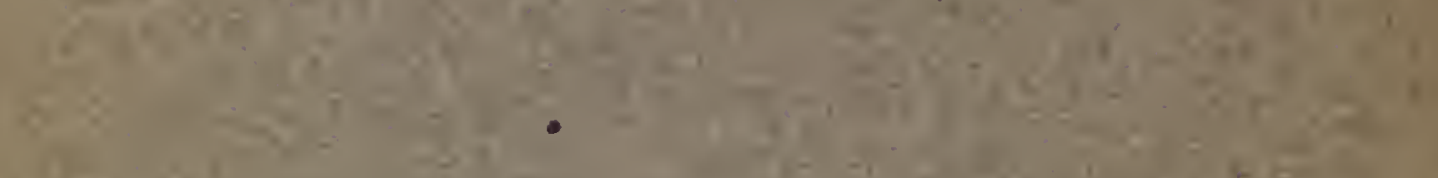

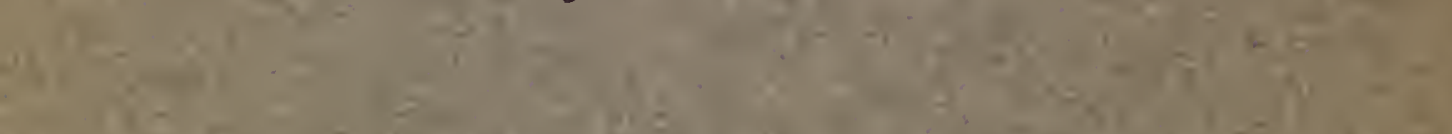

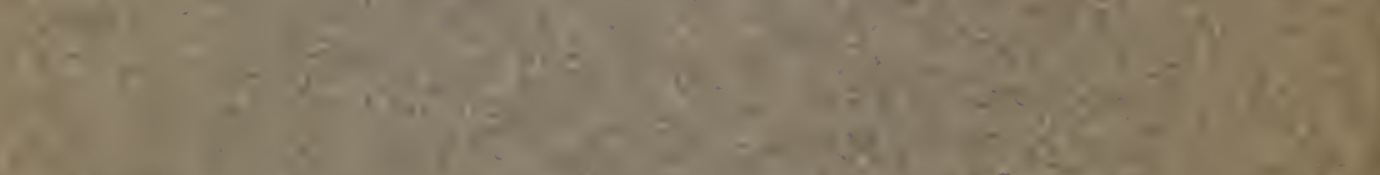

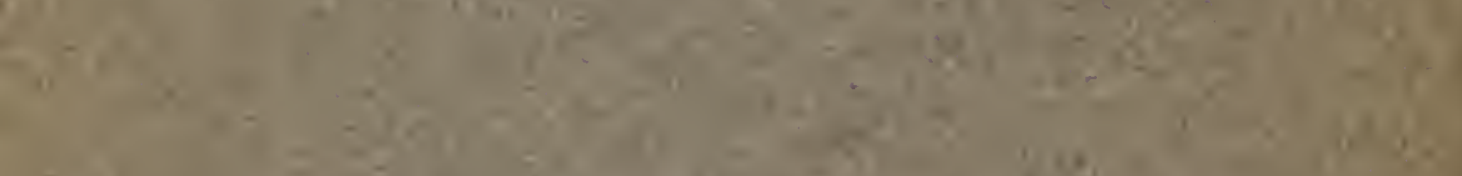

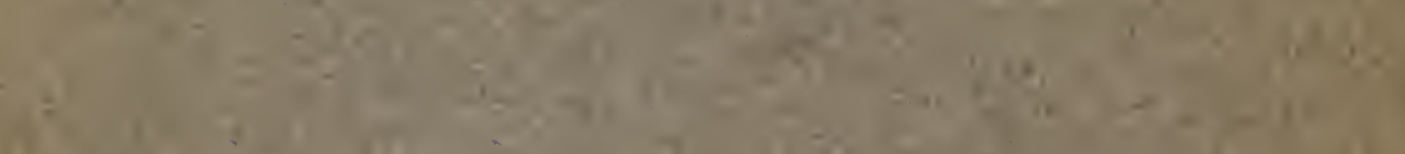

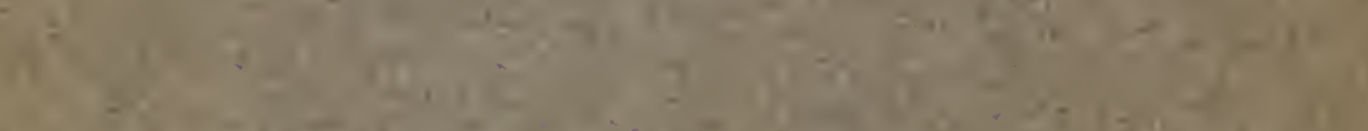

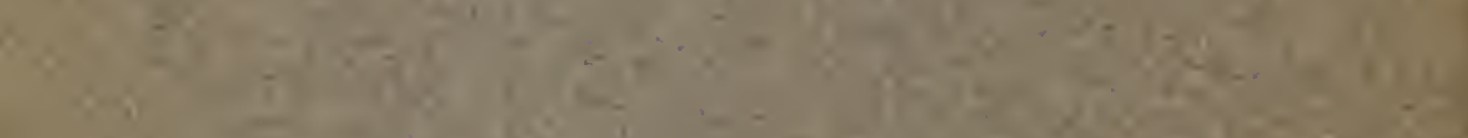

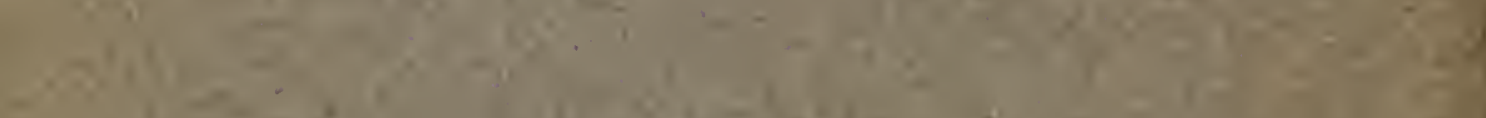

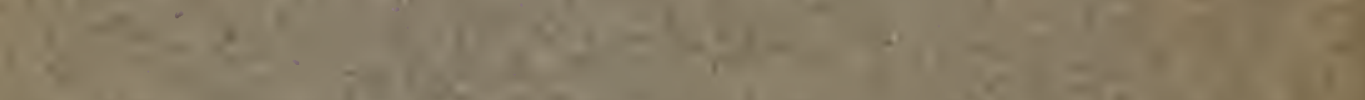

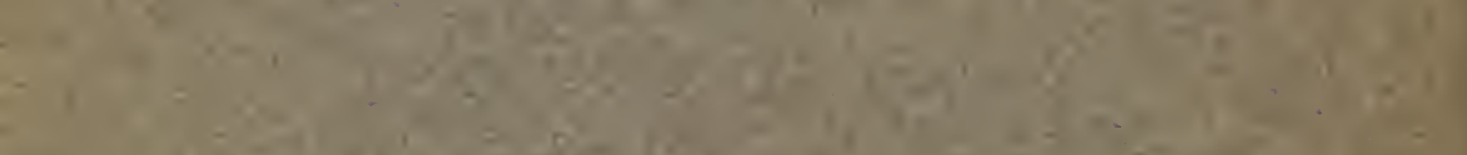

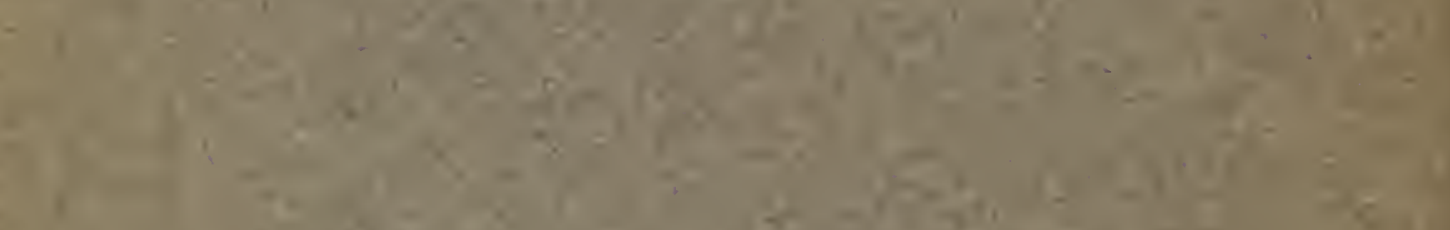

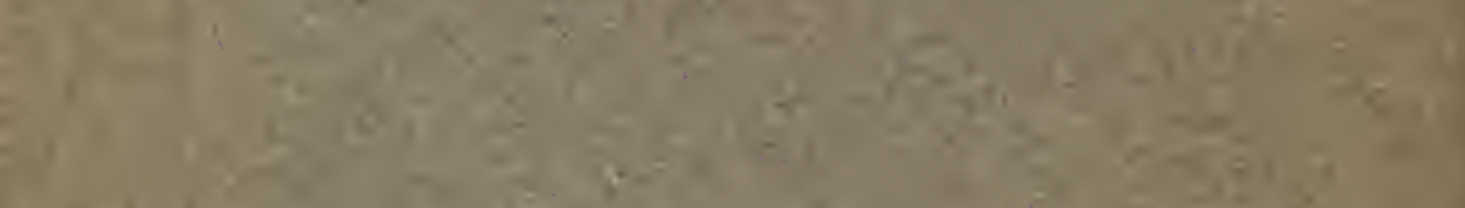

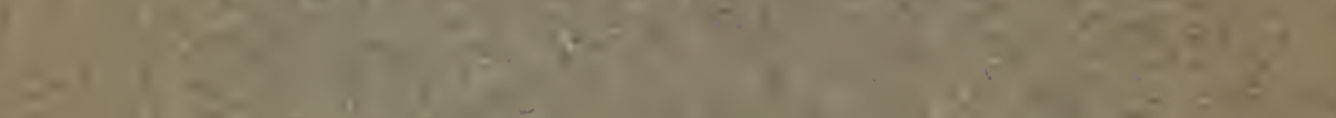

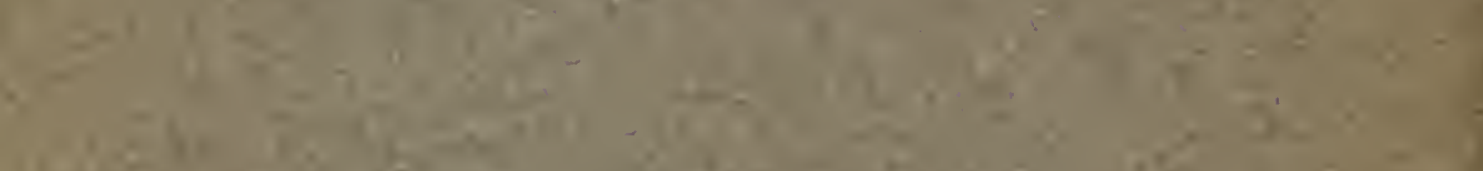

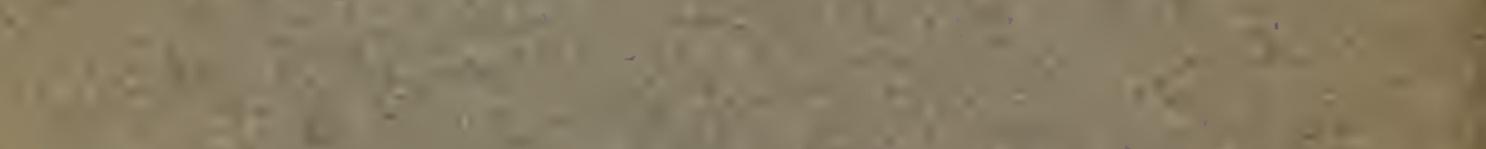

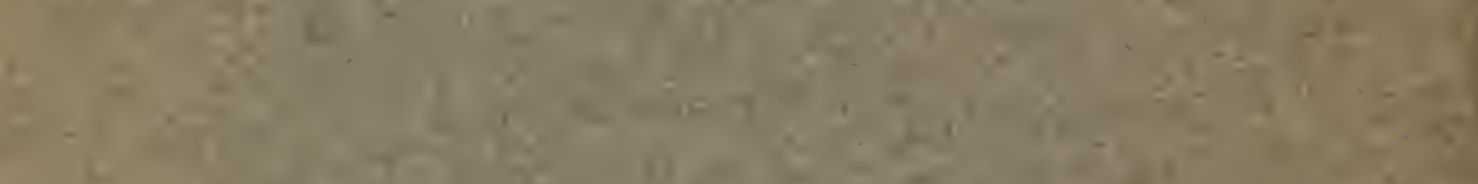

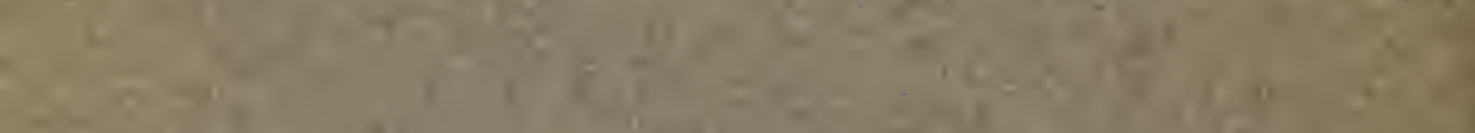

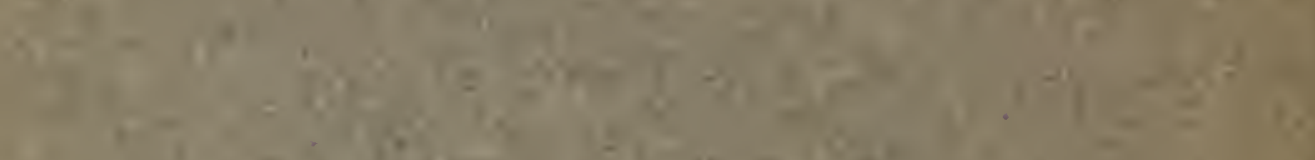


onล $19^{\circ} \gamma$

$\pm 1$ 
